\title{
Biocontrol of some toxigenic fungal strains and their toxins production using some spices
}

\author{
Zohri A.A ${ }^{1}$, Saber S.M ${ }^{2}$, Youssef M.S ${ }^{2}$, Marwa Abdel-Kareem M. ${ }^{2}$ \\ ${ }^{1}$ Botany \& Microbiology Department, Faculty of Science, Assiut University, Egypt. \\ ${ }^{2}$ Botany Department, Faculty of Science, Sohag University, Egypt.
}

Rec. 1 Mar, 2014 Accept. 3 Apr, 2014

\begin{abstract}
The inhibitory effect of nine spices against some toxigenic fungi and their ability for producing toxins in order to use as a biocontrol agents as food additieves were tested on five local isolates isolated from different Egyptian food sources and six isolates obtained from CBS (Central Bureau voor Schimmelcultures), Holland. Clove was found to be the most effective spice which completely inhibited the growth and mycotoxins production at concentration of $0.5,1 \%(\mathrm{w} / \mathrm{v})$ respectively and completely inhibited fungal count and mycotoxins production when used as food additive at concentration of $1 \%(\mathrm{w} / \mathrm{v})$. Black pepper, cinnamon, spearmint, cumin, thyme and anise were inhibited the growth of most fungal isolates and completely inhibited mycotoxins production at concentration of $1 \%$.
\end{abstract}

Key words: toxigenic fungi, mycotoxins, spices.

\section{Introduction}

Many fungi producing mycotoxins are frequent contaminants of foodstuffs and, when conditions are favorable for growth, they grow and produce mycotoxins. Thus it is obvious that if the growth of toxigenic fungi can be prevented subsequent contamination with mycotoxins will also be prevented. The use of many of the available physical and chemical methods for preserving foods from contamination with toxigenic fungi and their toxins is restricted due to problems concerning safety issues, possible losses in the nutritional quality of treated foods and coupled with limited efficacy and cost implications (Köhl et al., 2011). However, in most countries chemical and physical preservation are not permitted in foods. The need thus arises for natural preservatives that could be used for semi processed and processed foods. Currently the global trend is turned to safer and ecofriendly alternative approaches (Mari et al., 2007; Sharma et al., 2009). One of these possibilities is the use of spices to control mycotoxigenic fungi.

Spices have been added to foods since ancient times, not only as flavoring agents, but also as folk medicine and food preservatives (Beuchat, 1994; Cutler, 1995). In addition to imparting characteristic flavors, certain spices prolong the storage life of foods by preventing rancidity through their antioxidant activity or through bacteriostatic or bactericidal activity (Beuchat and Golden, 1989). Spices are generally recognized to be safe, either because of their traditional use without any documented detrimental impact or because of dedicated toxicological studies (Smid and Gorris, 1999). Being natural foodstuffs, spices appeal to many consumers who question the safety of synthetic food additives. Some spices used today are valued for their antimicrobial activities and medicinal effects in addition to their flavor and fragrance qualities. The extracts of many spices have become popular in recent years and attempts to characterize their bioactive principles have gained momentum for varied pharmaceutical and food processing applications.

Today, increasing the interest in identifying naturally occurring materials such as spices for limit or prevent the toxigenic fungi growth and/ or their ability to produce mycotoxin. Thus, this study was conducted to determine whether spices with high activity would inhibit fungal growth and mycotoxin production of selected 11 toxigenic fungal isolates.

\footnotetext{
* Corresponding author: 
Materials and Methods Selection of toxigenic fungi:

A total of 11 toxigenic fungal isolates were selected for studying the biocontrol activities of some spices on their growth and toxins formation. The toxigenic selected fungal isolates were 5 isolates which were isolated from different food sources in Sohag Governorate, Egypt and recorded as highly toxin producers (local isolates), these isolates were Aspergillus flavus 30 (Aflatoxin $\mathrm{B}_{1}, \mathrm{~B}_{2}$, $\mathrm{G}_{1}$ and $\mathrm{G}_{2}$ producer), A. ochraceus 76 (Ochratoxins A, B), Aspergillus nidulans 69 (Sterigmatocystin), Penicillium digitatum 131 (Patulin) and Alternaria alternata 5 (Alternariol). Other six highly toxigenic fungal isolates were purchased from CBS (Centraal Bureau voor Schimmelcultures), Fungal Biodiversity Center of Holland and were used as a standard isolates. These isolates were Aspergillus parasiticus CBS 571.65 (Aflatoxin $\mathrm{B}_{1}, \mathrm{~B}_{2}, \mathrm{G}_{1}$ and $\mathrm{G}_{2}$ ), $A$. ochraceus CBS 589.68 (Ochratoxin A), Penicillium griseofulvum CBS 589.68 (Patulin), P. scabrosum CBS 530.97 (Fumagillin), Fusarium equiseti CBS 406.86 (Zearalenone) and Phaeosphaeria nodorum CBS 438.87 (Alternariol).

\section{Preparation of spices:}

Nine types of spices (anise, black pepper, caraway, cinnamon, clove, cumin, ginger, spearmint and thyme) were collected and dried in hot oven for 4 days at $65^{\circ} \mathrm{C}$. The dried spices were ground to powder and used for the biocontrol treatment.

\section{The effect of spices on growth of toxigenic fungal isolates:}

This was done according to the method described by Mabrouk and El-Shayeb, 1981). with some modification as follow: the dried spices were used in three concentrations $(1 \mathrm{~g}$, $5 \mathrm{~g}$ and $10 \mathrm{~g} / \mathrm{L}$ medium). Fungi were grown in Petri dishes on the potato dextrose agar medium with one of the three different concentrations of one of the tested 9 spices, dishes were incubated at $28^{\circ} \mathrm{C}$. The effect of the spices was detected by measuring the diameter of fungal colonies after four days of incubation and by comparing them to the control culture (dishes with fungi but without spices).

\section{The effect of spices on mycotoxins production:}

Six spices (cumin, caraway, anise, cinnamon, thyme and cloves) which proved to be more effective on fungal growth were used in this experiment. Each individual fungal isolate was cultivated on potato dextrose liquid medium contains $0.5 \mathrm{~g}$ of one of the spices under examination. Erlenmeyer flasks of $250 \mathrm{ml}$ capacity were used. Each flask contained $50 \mathrm{ml}$ medium. The flasks were sterilized at 1.5 atmospheres for 20 minutes and inoculated after cooling with two $\mathrm{ml}$ of the inoculum suspension of 10 days old culture of the pure organism. The cultures were incubated at $28 \pm 2^{\circ} \mathrm{C}$ for 10 days. At the end of incubation period the content of each flask (medium + mycelium) were homogenized for five minutes in a high speed blender (16000 rpm) with $100 \mathrm{ml}$ chloroform. The chloroform extracts were washed with equal volume of distilled water, dried over anhydrous sodium sulphate, filtered then concentrated to near dryness. Mycotoxin levels were detected using thin layer chromatography (Scott et al., 1970; Gimeno, 1979; El-kady and Moubasher, 1982).

\section{The effect of clove on fungal counts and toxin formation in some foodstuffs:}

Clove $(10 \mathrm{~g} / \mathrm{l})$ which proved to be the effective spice on both growth and toxin production by the 11 toxigenic fungi in the previous experiment was tested for the ability to affect the counts of fungi in different 5 foodstuffs (apple, guava juice, rice, wheat and maize). The counts of each fungal species in selected foodstuffs were detected and compared with the counts of the same fungal species in each foodstuff, individually, in presence of the clove mixed with the foodstuffs. On the other hand, the effects of clove $(10 \mathrm{~g} / \mathrm{l})$ on the production of aflatoxin, ochratoxin and zearalenone by $A$. flavus 30, A. ochraceus 76 and $F$. equiseti CBS 406.86, respectively, in three foodstuffs (guava juice, wheat and maize) were examined. The toxins formed in each of the three selected foodstuffs (free from mycotoxins), individually, by each of the three selected toxigenic fungi, individually, were detected and compared with those recorded in presence of clove.

\section{Results and Discussion}

The inhibitory effect of the nine spices was been tested on the growth of the six 
standard and five local toxigenic fungal isolates. The results (Tables, $1 \& 2$ ) showed that clove was the strongest antifungal spice against the growth at concentration of $0.5 \%$ $(5 \mathrm{~g} / \mathrm{l})$, which is completely, inhibited the growth of all tested isolates. Black pepper, cinnamon and spearmint showed less inhibitory effect, which are completely inhibited the growth of all tested fungal isolates at concentration of $1 \%(10 \mathrm{~g} / \mathrm{l})$. At the same concentration of $1 \%$, we found that Cumin, thyme and anise spices have selective inhibitory effect of $100 \%$ on the tested fungal isolates. On the other hand, caraway and ginger exhibited highly reduction of fungal growth at concentration of $1 \%$. Antimicrobial properties of spices have been documented in recent years and interest continues to the present (El Shami et al., 1985; Akgul and Kivanç, 1988; Cosentino et al., 1999; Domans and Deans, 2000; Ristori et al., 2002; RadhakrishananSridhar and Velusamy-Rajaopal, 2003; Souza et al., 2005; Bokhari, 2007; Bokhari and Aly, 2009). Many spices and herbs, such as cloves (Hitokoto et al., 1980). basil and cinnamon (Leontopoulos et al., 2003). garlic (Benkeblia, 2004). thyme (Rasooli et al., 2006). cassia and sweet basil (Atanda et al., 2007) have been reported to inhibit toxigenic and food-borne fungi. (Juglal et al., 2002). studied the effectiveness of nine spices to control the growth of mycotoxins producing moulds and noted that clove and cinnamon were able to prevent the growth of Aspergillus parasiticus and Fusarium moniliforme, (Bokhari, 2007). reported that among 10 kinds of spices, clove was the most effective against three isolates of toxigenic fungi namely $A$. flavus, $A$. versicolor and $P$. citrinum which produced aflatoxins, sterigmatocystin and citrinin, respectively. (Thyagaraja and Hosono, 1996). assayed the ability of pepper and cumin to inhibit food spoilage moulds Rhizopus azygosporus, Mucor dimorphosphorous, Penicillium commune and Fusarium solani. (Schmitz et al., 1993). reported that at $2 \%$ concentration cinnamon is active against the food-borne fungi Trichoderma harzianum, Alternaria alternata, Fusarium culmorum, Aspergillus versicolor, Cladosporium cladosporoides and Penicillium citrinum.
The results in tables $(3 \& 4)$ indicated that production of all mycotoxins were completely inhibited in presence of $1 \%(\mathrm{w} / \mathrm{v})$ each spice with only exception in case of Alternaria alternata 5. Mycotoxin production by A. alternata 5 was completely inhibited in presence of cumin, clove, cinnamon, anise and caraway and partially reduced (60\% inhibition) in presence of thyme. (Bokhari, 2007). reported that five spices completely inhibited both aflatoxin and sterigmatocystin production, the five kinds of spices was black pepper, peppermint, cardamom, cumin and marjoram. It was found that cinnamon, cloves or both inhibited both growth (50\%) and Aflatoxin $\mathrm{G}_{1}$ production (100\%) of $A$. flavus (Bokhari and Aly, 2009). (Farag et al., 1989). indicated that caraway at $0.8 \mathrm{mg} / \mathrm{ml}$ exhibits antifungal activity against $A$. parasiticus and their aflatoxin production was completely inhibited. (Dubey et al., 1991). reported that cumin was fungistatic at lower doses but at higher doses is fungicidal against aflatoxin-producing strains of $A$. flavus and A. parasiticus. (Mostafa, 1990). examined the inhibitory effects of 24 commercial spices on toxin production by two toxigenic Aspergillus (A. flavus, A. versicolor) and two Penicillium ( $P$. citrinum and $P$. corylophilum) and found that chinese cassia, cinnamon, clove and thyme completely inhibited the fungal growth of the tested fungal isolates. The antitoxigenic potential of the spices was tested against ochratoxin producing strain of A. ochraceus, Clove completely inhibited the mycelial growth of the fungi A. ochraceus. Cinnamon and anis inhibited the synthesis of ochratoxin starting from the concentration of $3 \%$ and mint starting from 4\% (Bugno et al., 2006).

Clove was tested as the best biocontrol spice against the fungal counts of five food sources and toxin production by three fungal isolates on three food types (Table, 5). The results appeared that clove spice completely inhibited the growth of all fungi in the five samples of food sources and completely inhibited aflatoxin, ochratoxin and zearalenone production on guava juice. Zearalenone production on wheat was completely inhibited by clove while aflatoxin and ochratoxin production were reduced by $60 \%$.In the other hand, aflatoxin production 
on maize was only reduced by $40 \%$ in the presence of clove while ochratoxin and zearalenone production were completely inhibited. (Juglal et al., 2002). reported that clove markedly reduced the aflatoxin synthesis in infected grains. These findings could be useful for rural communities to prevent the synthesis of fungal toxins in contaminated grains by simple measures. (Awuah and Ellis, 2002). reported the effective use of powders of leaves of cloves combination with some packaging materials to protect groundnut kernels artificially inoculated with $A$. parasiticus.

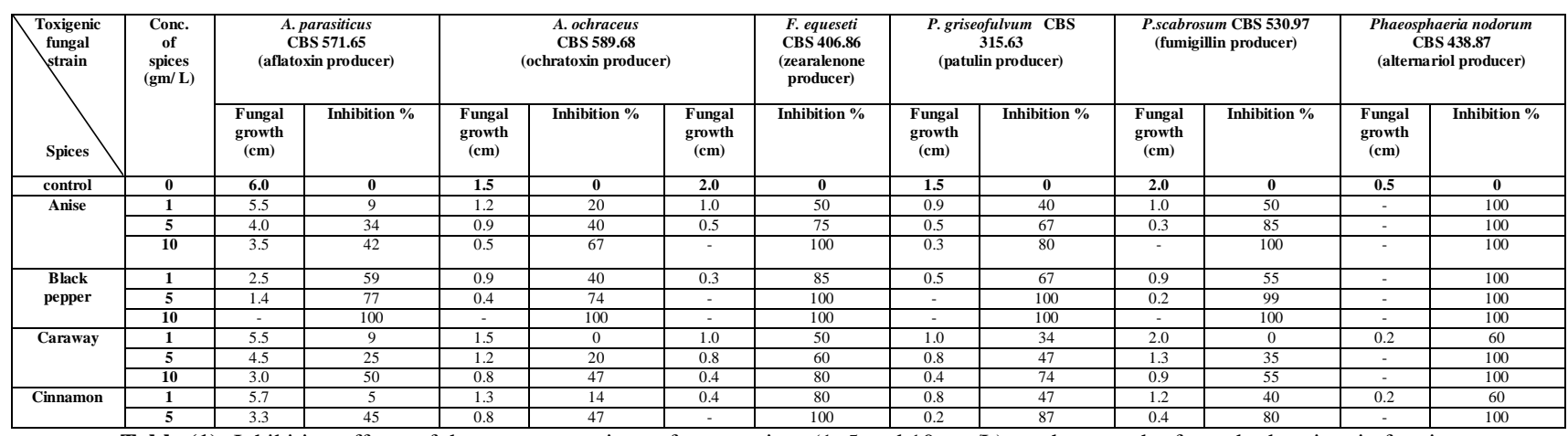

Table (1): Inhibition effects of three concentrations of some spices (1, 5 and $10 \mathrm{gm} / \mathrm{L})$ on the growth of standard toxigenic fungi (growth diameter measured by $\mathrm{cm}$ after 4 days of incubation on PDA medium at $28{ }^{\circ} \mathrm{C}$ ).

\begin{tabular}{|c|c|c|c|c|c|c|c|c|c|c|c|c|c|}
\hline \multirow{2}{*}{$\begin{array}{c}\text { Toxigenic } \\
\text { fungal } \\
\text { styain } \\
\text { Spices } \\
\end{array}$} & \multirow[t]{2}{*}{ 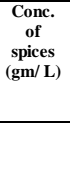 } & \multicolumn{2}{|c|}{$\begin{array}{c}\text { A. parasiticus } \\
\text { CBS 571.65 } \\
\text { (aflatoxin producer) }\end{array}$} & \multicolumn{2}{|c|}{$\begin{array}{c}\text { A. ochraceus } \\
\text { CBS 589.68 } \\
\text { (ochratoxin producer) }\end{array}$} & \multicolumn{2}{|c|}{$\begin{array}{l}\text { F. equeseti CBS } 406.86 \\
\text { (zearalenone producer) }\end{array}$} & \multicolumn{2}{|c|}{$\begin{array}{c}\text { P. griseofulvum CBS } \\
315.63 \\
\text { (patulin producer) }\end{array}$} & \multicolumn{2}{|c|}{$\begin{array}{l}\text { P.scabrosum CBS 530.97 } \\
\text { (fumigillin producer) }\end{array}$} & \multicolumn{2}{|c|}{$\begin{array}{c}\text { Phaeosphaeria nodorum } \\
\text { CBS 438.87 } \\
\text { (alternariol producer) }\end{array}$} \\
\hline & & $\begin{array}{c}\begin{array}{c}\text { Fungal } \\
\text { growth } \\
(\mathrm{cm})\end{array} \\
\text {. }\end{array}$ & Inhibition \% & $\begin{array}{c}\begin{array}{c}\text { Fungal } \\
\text { growth } \\
\text { (cm) }\end{array}\end{array}$ & Inhibition \% & $\begin{array}{c}\begin{array}{c}\text { Fungal } \\
\text { growth } \\
(\mathbf{c m})\end{array}\end{array}$ & Inhibition \% & $\begin{array}{c}\begin{array}{c}\text { Fungal } \\
\text { growth } \\
(\mathbf{c m})\end{array}\end{array}$ & Inhibition \% & $\begin{array}{c}\text { Fungal } \\
\text { growth } \\
\text { (cm) }\end{array}$ & Inhibition \% & $\begin{array}{c}\text { Fungal } \\
\text { growth } \\
(\mathrm{cm})\end{array}$ & Inhibition \% \\
\hline control & 0 & 6.0 & 0 & 1.5 & 0 & 2.0 & 0 & 1.5 & 0 & 2.0 & 0 & 0.5 & 0 \\
\hline $\begin{array}{l}\text { Cinnamon } \\
\end{array}$ & 10 & & 100 & 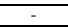 & 100 & & 100 & - & 100 & & 100 & - & 100 \\
\hline \multirow[t]{3}{*}{ Clove } & 1 & 3.5 & 42 & 0.8 & 47 & 0.4 & 74 & 0.5 & 75 & 0.4 & 80 & - & 100 \\
\hline & 5 & - & 100 & - & 100 & - & 100 & - & 100 & - & 100 & - & 100 \\
\hline & 10 & - & 100 & - & 100 & - & 100 & - & 100 & - & 100 & - & 100 \\
\hline \multirow[t]{3}{*}{ Cumin } & 1 & 5.3 & 12 & 1.2 & 20 & 0.7 & 65 & 0.9 & 40 & 1.7 & 15 & - & 100 \\
\hline & 5 & 3.8 & 37 & 0.8 & 47 & 0.2 & 99 & 0.5 & 67 & 1.0 & 50 & - & 100 \\
\hline & 10 & 1.5 & 75 & - & 100 & & 100 & 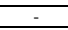 & 100 & & 100 & - & 100 \\
\hline \multirow[t]{3}{*}{ Ginger } & 1 & 5.5 & 9 & 1.5 & 0 & 1.0 & 50 & 0.8 & 47 & 1.7 & 15 & 0.4 & 20 \\
\hline & 5 & 4.0 & 34 & 0.9 & 40 & 0.8 & 60 & 0.6 & 60 & 1.4 & 30 & 0.2 & 60 \\
\hline & 10 & 3.5 & 42 & 0.4 & 74 & 0.5 & 75 & 0.4 & 74 & 1.0 & 50 & - & 100 \\
\hline \multirow[t]{2}{*}{ Spearmint } & 1 & 5.3 & 12 & 1.4 & 7 & 1.0 & 50 & 0.9 & 40 & 1.3 & 35 & - & 100 \\
\hline & 5 & 2.5 & 59 & 0.8 & 47 & 0.4 & 80 & 0.3 & 80 & 0.5 & 75 & - & 100 \\
\hline
\end{tabular}

Table (1): Continued.

\begin{tabular}{|c|c|c|c|c|c|c|c|c|c|c|c|c|c|}
\hline \multirow{2}{*}{$\begin{array}{c}\text { Toxigenic } \\
\text { fungal } \\
\text { styain } \\
\text { Spice } \\
\end{array}$} & \multirow{2}{*}{$\begin{array}{l}\text { Conc. } \\
\text { of } \\
\text { spices } \\
(\mathrm{gm} / \mathrm{L})\end{array}$} & \multicolumn{2}{|c|}{$\begin{array}{c}\text { A. parasiticus } \\
\text { CBS 571.65 } \\
\text { (aflatoxin producer) }\end{array}$} & \multicolumn{2}{|c|}{$\begin{array}{c}\text { A. ochraceus } \\
\text { CBS 589.68 } \\
\text { (ochratoxin producer) }\end{array}$} & \multicolumn{2}{|c|}{$\begin{array}{l}\text { F. equeseti CBS } 406.86 \\
\text { (zearalenone producer) }\end{array}$} & \multicolumn{2}{|c|}{$\begin{array}{c}\text { P. griseofulvum CBS } \\
315.63 \\
\text { (patulin producer) }\end{array}$} & \multicolumn{2}{|c|}{$\begin{array}{l}\text { P.scabrosum CBS 530.97 } \\
\text { (fumigillin producer) }\end{array}$} & \multicolumn{2}{|c|}{$\begin{array}{c}\text { Phaeosphaeria nodorum } \\
\text { CBS 438.87 } \\
\text { (alternariol producer) }\end{array}$} \\
\hline & & $\begin{array}{l}\text { Fungal } \\
\text { growth } \\
(\mathrm{cm})\end{array}$ & Inhibition \% & $\begin{array}{l}\begin{array}{l}\text { Fungal } \\
\text { growth } \\
\text { (cm) }\end{array}\end{array}$ & Inhibition \% & $\begin{array}{l}\text { Fungal } \\
\text { growth } \\
\text { (cm) }\end{array}$ & Inhibition \% & $\begin{array}{l}\begin{array}{l}\text { Fungal } \\
\text { growth } \\
(\mathrm{cm})\end{array}\end{array}$ & Inhibition \% & $\begin{array}{l}\text { Fungal } \\
\text { growth } \\
(\mathrm{cm})\end{array}$ & Inhibition \% & $\begin{array}{l}\text { Fungal } \\
\text { growth } \\
(\mathrm{cm})\end{array}$ & Inhibition \% \\
\hline control & 0 & 6.0 & 0 & 1.5 & 0 & 2.0 & 0 & 1.5 & 0 & 2.0 & 0 & 0.5 & 0 \\
\hline Spearmint & 10 & & 100 & & 100 & & 100 & & 100 & & 100 & - & 100 \\
\hline \multirow[t]{3}{*}{ Thyme } & 1 & 5.0 & 17 & 1.4 & 7 & 0.5 & 75 & 1.0 & 34 & 1.3 & 35 & - & 100 \\
\hline & 5 & 3.0 & 50 & 0.3 & 80 & & 100 & 0.5 & 67 & 0.8 & 60 & - & 100 \\
\hline & 10 & 2.0 & 67 & - & 100 & - & 100 & 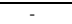 & 100 & - & 100 & - & 100 \\
\hline
\end{tabular}

Table (1): Continued.

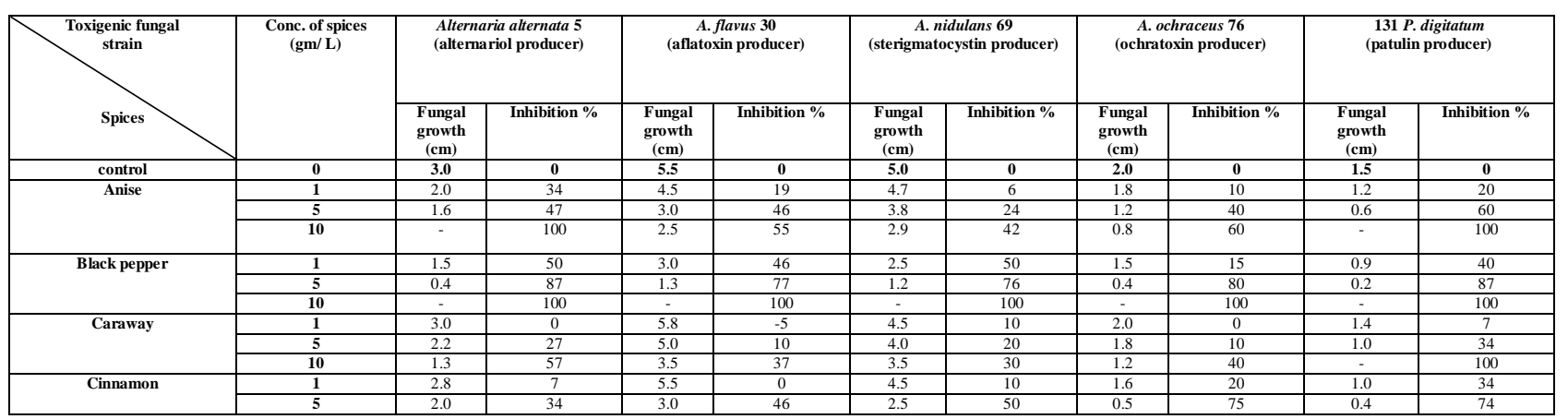

Table (2): Inhibition effects of three concentrations of some spices $(1,5$ and $10 \mathrm{gm} / \mathrm{L})$ on the growth of local toxigenic fungi (growth diameter measured by $\mathrm{cm}$ after 4 days of incubation on PDA medium at $28^{\circ} \mathrm{C}$ ). 


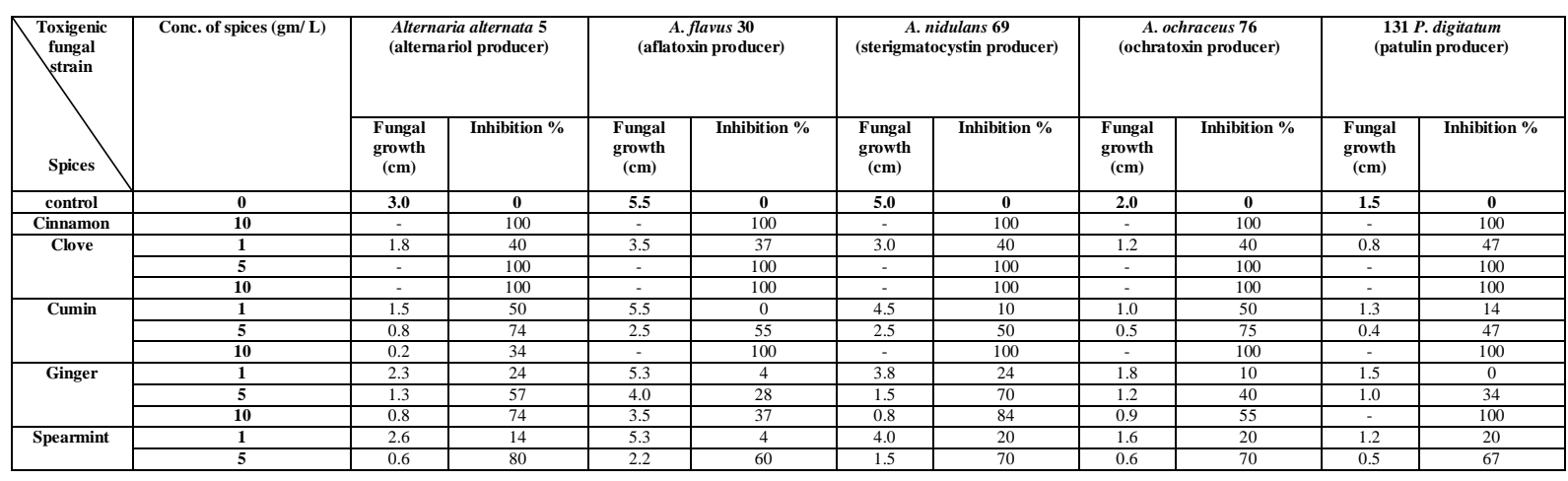

Table (2): Continued.

\begin{tabular}{|c|c|c|c|c|c|c|c|c|c|c|c|}
\hline \multirow{2}{*}{$\overbrace{\text { Spices }} \begin{array}{c}\text { Toxigenic } \\
\text { fungal } \\
\text { strain }\end{array}$} & \multirow[t]{2}{*}{ Conc. of spices $(\mathrm{gm} / \mathrm{L})$} & \multicolumn{2}{|c|}{$\begin{array}{l}\text { Alternaria alternata } 5 \\
\text { (alternariol producer) }\end{array}$} & \multicolumn{2}{|c|}{$\begin{array}{c}\text { A. flavus 30 } \\
\text { (aflatoxin producer) }\end{array}$} & \multicolumn{2}{|c|}{$\begin{array}{c}\text { A. nidulans } 69 \\
\text { (sterigmatocystin producer) }\end{array}$} & \multicolumn{2}{|c|}{$\begin{array}{c}\text { A. ochraceus 76 } \\
\text { (ochratoxin producer) }\end{array}$} & \multicolumn{2}{|c|}{$\begin{array}{l}131 \underset{\text { digitatum }}{P .} \\
\text { (patulin producer) }\end{array}$} \\
\hline & & $\begin{array}{c}\text { Fungal } \\
\text { growth } \\
(\mathbf{c m})\end{array}$ & Inhibition \% & $\begin{array}{c}\text { Fungal } \\
\text { growth } \\
\text { (cm) }\end{array}$ & Inhibition \% & $\begin{array}{c}\text { Fungal } \\
\text { growth } \\
(\mathrm{cm})\end{array}$ & Inhibition \% & $\begin{array}{l}\text { Fungal } \\
\text { growth } \\
\text { (cm) }\end{array}$ & Inhibition \% & $\begin{array}{c}\text { Fungal } \\
\text { growth } \\
(\mathbf{c m})\end{array}$ & Inhibition \% \\
\hline control & 0 & 3.0 & 0 & 5.5 & 0 & 5.0 & 0 & 2.0 & 0 & 1.5 & 0 \\
\hline Spearmint & 10 & & 100 & -1 & 100 & 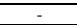 & 100 & 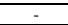 & 100 & & 100 \\
\hline \multirow[t]{3}{*}{ Thyme } & 1 & 2.7 & 10 & 5.3 & 4 & 4.0 & 20 & 1.8 & 10 & 1.0 & 34 \\
\hline & 5 & - & 100 & 2.3 & 42 & 2.0 & 60 & 0.5 & 75 & 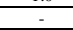 & 100 \\
\hline & 10 & - & 100 & 2.0 & 64 & & 100 & & 100 & - & 100 \\
\hline
\end{tabular}

Table (2): Continued.

\begin{tabular}{|c|c|c|c|c|c|c|c|c|c|c|c|c|}
\hline \multirow{2}{*}{$\begin{array}{c}\text { Toxigenic } \\
\text { fungal } \\
\text { strain }\end{array}$} & \multicolumn{2}{|c|}{$\begin{array}{c}\text { A.parasiticus } \\
\text { CBS 571.65 } \\
\text { (aflatoxin producer) }\end{array}$} & \multicolumn{2}{|c|}{$\begin{array}{c}\text { A. ochraceus } \\
\text { CBS 589.68 } \\
\text { (ochratoxin producer) }\end{array}$} & \multicolumn{2}{|c|}{$\begin{array}{l}\begin{array}{l}\text { F. equeseti CBS } 406.86 \\
\text { (zearalenone producer) }\end{array} \\
\text { (a) }\end{array}$} & \multicolumn{2}{|c|}{$\begin{array}{c}\text { P. griseofulvum CBS } \\
315.63 \\
\text { (patulin producer) }\end{array}$} & \multicolumn{2}{|c|}{$\begin{array}{l}\text { P.scabrosum CBS 53097 } \\
\text { (fumigillin producer) }\end{array}$} & \multicolumn{2}{|c|}{$\begin{array}{c}\text { Phaeosphaeria nodorum } \\
\text { CBS } 438.87 \\
\text { (alternariol producer) }\end{array}$} \\
\hline & $\begin{array}{l}\begin{array}{c}\text { Toxin } \\
\text { level }\end{array} \\
\end{array}$ & $\begin{array}{c}\text { Inhibition \% } \\
\text { of toxin } \\
\text { production }\end{array}$ & $\begin{array}{l}\text { Toxin } \\
\text { level }\end{array}$ & $\begin{array}{c}\text { Inhibition \% } \\
\text { of toxin } \\
\text { production }\end{array}$ & $\begin{array}{l}\text { Toxin } \\
\text { level }\end{array}$ & $\begin{array}{c}\text { Inhibition \% } \\
\text { of toxin } \\
\text { production }\end{array}$ & $\begin{array}{l}\text { Toxin } \\
\text { level }\end{array}$ & $\begin{array}{c}\text { Inhibition \% of } \\
\text { toxin } \\
\text { production }\end{array}$ & $\begin{array}{l}\text { Toxin } \\
\text { level }\end{array}$ & $\begin{array}{c}\text { Inhibition \% } \\
\text { of toxin } \\
\text { production }\end{array}$ & $\begin{array}{l}\text { Toxin } \\
\text { level }\end{array}$ & $\begin{array}{c}\text { Inhibition \% } \\
\text { of toxin } \\
\text { production }\end{array}$ \\
\hline control & +5 & 0 & +5 & 0 & +5 & 0 & +5 & 0 & +5 & 0 & +5 & 0 \\
\hline Anise & 0 & 100 & 0 & 100 & 0 & 100 & 0 & 100 & 0 & 100 & 0 & 100 \\
\hline Caraway & 0 & 100 & 0 & 100 & 0 & 100 & 0 & 100 & 0 & 100 & 0 & 100 \\
\hline$\underset{\mathbf{n}}{\text { Cinnamo }}$ & 0 & 100 & 0 & 100 & 0 & 100 & 0 & 100 & 0 & 100 & 0 & 100 \\
\hline Clove & 0 & 100 & 0 & 100 & 0 & 100 & 0 & 100 & 0 & 100 & 0 & 100 \\
\hline Cumin & 0 & 100 & 0 & 100 & 0 & 100 & 0 & 100 & 0 & 100 & 0 & 100 \\
\hline Thyme & 0 & 100 & 0 & 100 & 0 & 100 & 0 & 100 & 0 & 100 & 0 & 100 \\
\hline
\end{tabular}

Table (3): Inhibition effect (\%) of some spices $(0.5 \mathrm{gm} / 50 \mathrm{ml}$ medium) on mycotoxins production by standard toxigenic fungi grown on potato- dextrose liquid medium supplemented with the spice at $28{ }^{\circ} \mathrm{C}$ for 10 days.

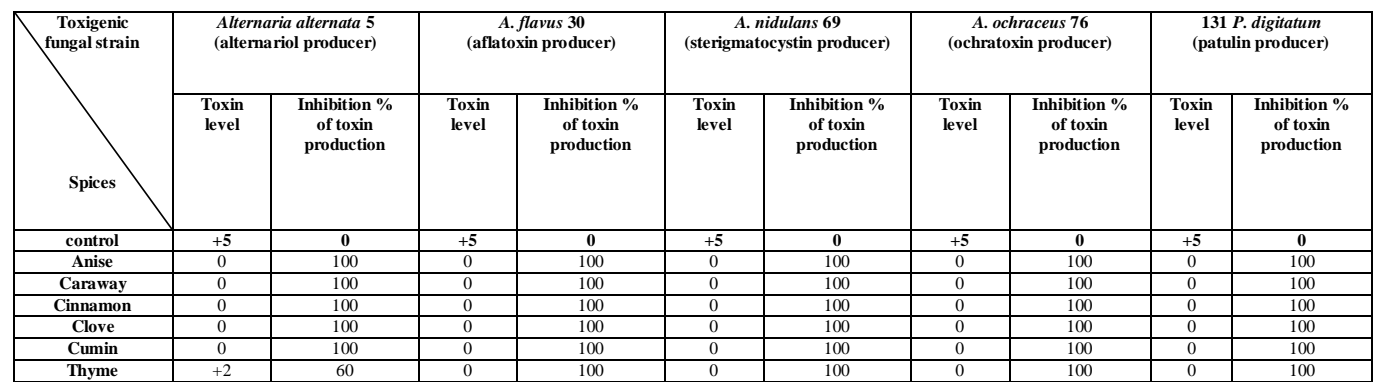

Table (4): Inhibition effect (\%) of some spices $(0.5 \mathrm{gm} / 50 \mathrm{ml}$ medium) on mycotoxins production by local toxigenic fungi grown on potato- dextrose liquid medium supplemented with the spice at $28^{\circ} \mathrm{C}$ for 10 days. 


\begin{tabular}{|c|c|c|c|c|c|c|c|c|}
\hline \multirow{2}{*}{$\overbrace{\text { Food sources }}^{\text {Biocontrol agent }}$} & \multicolumn{2}{|c|}{ Control } & \multicolumn{2}{|c|}{ Clove } & \multicolumn{3}{|c|}{ Control } & \multirow[t]{2}{*}{ Clove } \\
\hline & \multirow{2}{*}{$\begin{array}{c}\text { Fungi } \\
\\
\text { Aspergillusflavus } \\
\text { A. niger } \\
\text { A. terreus } \\
\text { Penicillium rubrum }\end{array}$} & \multirow{2}{*}{$\begin{array}{c}\text { Av. No. } \\
2 \\
3 \\
2 \\
3\end{array}$} & \multirow{2}{*}{$\begin{array}{c}\text { Fungi } \\
- \\
- \\
-\end{array}$} & \multirow{2}{*}{$\begin{array}{c}\text { Av. No. } \\
- \\
\vdots \\
\end{array}$} & - -ve & \multicolumn{2}{|l|}{$+\mathrm{ve}$} & \\
\hline & & & & & - & N.T. & - & - \\
\hline \multirow[t]{3}{*}{$\begin{array}{l}\text { Guava juice } \\
\end{array}$} & \multirow{3}{*}{$\begin{array}{c}\text { Aspergillus flavus } \\
\text { A. niger } \\
\text { Rhizopus nigricans }\end{array}$} & \multirow{3}{*}{$\begin{array}{c}7 \\
8 \\
20\end{array}$} & \multirow{3}{*}{ : } & \multirow{3}{*}{$\overline{-}$} & \multirow[t]{3}{*}{. } & A. flavus (aflatoxin) & +4 & $100 \%$ \\
\hline & & & & & & A. ochraceus (ochratoxin) & +3 & $100 \%$ \\
\hline & & & & & & F. equiseti (zearalenone) & +4 & $100 \%$ \\
\hline Rice & $\begin{array}{c}\text { Aspergillus flavus } \\
\text { A. ochraceus } \\
\text { Fusarium solani } \\
\text { Penicillium citrinum } \\
\text { Rhizopus oryzae } \\
\end{array}$ & $\begin{array}{l}4 \\
2 \\
4 \\
2 \\
2 \\
\end{array}$ & $\begin{array}{l}- \\
- \\
- \\
- \\
-\end{array}$ & $\begin{array}{l}- \\
- \\
- \\
- \\
-\end{array}$ & - & N.T. & - & - \\
\hline \multirow[t]{3}{*}{ Wheat } & \multirow{3}{*}{$\begin{array}{c}\text { Aspergillus flavus } \\
\text { A. sydowi } \\
\text { Penicillium } \\
\text { chrysogenum }\end{array}$} & \multirow{3}{*}{$\begin{array}{l}5 \\
3 \\
4\end{array}$} & \multirow{3}{*}{ : } & \multirow{3}{*}{ : } & \multirow[t]{3}{*}{ ? } & A. flavus (aflatoxin) & +5 & $60 \%$ \\
\hline & & & & & & A. ochraceus (ochratoxin) & +4 & $60 \%$ \\
\hline & & & & & & F. equiseti (zearalenone) & +5 & $100 \%$ \\
\hline \multirow[t]{3}{*}{ Maize } & \multirow{3}{*}{$\begin{array}{c}\text { Aspergillus flavus } \\
\text { Fusarium equesiti } \\
\text { Penicillium duclauxi }\end{array}$} & \multirow{3}{*}{$\begin{array}{c}4 \\
10 \\
4\end{array}$} & \multirow{3}{*}{$\begin{array}{l}- \\
-\end{array}$} & \multirow{3}{*}{$\begin{array}{l}- \\
-\end{array}$} & \multirow[t]{3}{*}{$\cdot$} & A. flavus (aflatoxin) & +5 & $40 \%$ \\
\hline & & & & & & A. ochraceus (ochratoxin) & +4 & $100 \%$ \\
\hline & & & & & & F. equiseti (zearalenone) & +4 & $100 \%$ \\
\hline
\end{tabular}

Table (5): Inhibitory effect of (1\%) clove on the fungal counts and toxins production in some food sources.

\section{References:}

Akgul, A. and Kivanç, M. (1988). Inhibitory effect of selected Turkish spices and oregano components on some foodborne fungi. International Journal of Food Microbiology. 6: 263-268.

Awuah, R.T. and Ellis, W.O. (2002). Effects of some groundnut packaging methods and protection with Ocimum and Syzygium powders on kernel infection by fungi. Mycopathologia. 154: 29-36.

Benkeblia, N. (2004). Antimicrobial activity of essential oil extracts of various onions (Allium cepa) and garlic (Allium sativum). LebensmittelWissenschaft und-TechnologieFood Science and Technology. 37: 263-268.

Beuchat, L.R. (1994). Antimicrobial properties of spices and their essential oils. In: Dillon, Y.M., Board, R.G. (Eds.), Natural Antimicrobial Systems and Food Preservation. $\quad \mathrm{CAB}$ International, Oxon, pp. 167-179.

Beuchat, L.R. and Golden, D.A. (1989). Antimicrobials occurring naturally in foods. Food Technology 43: 134-142.

Bokhari, F.M. (2007). Spices mycobiota and mycotoxins available in
Saudi Arabia and their abilities to inhibit growth of some toxigenic fungi. Mycobiology. 3: 47-53.

Bokhari, F.M. and Aly, M.M. (2009). Trials towards reduction of fungal growth and aflatoxin $\mathrm{G}_{1}$ production in Arabic coffee using different additives. African Journal of Food Science. 3: 68-76.

Bugno, A., Almodovar, A.A.B. and Pereira, T.C. (2006). Occurrence of toxigenic fungi in herbal drugs. Brazilian Journal of Microbiology. 37: 1-7.

Cosentino, S., Tuberoso, C.I.G., Pisano, B., Satta, M., Mascia, V., Arzedi, E. and Palmas, F. (1999). In-vitro antimicrobial activity and chemical composition of Sardinian Thymus essential oils. Letters in Applied Microbiology. 29: 130-135.

Cutler, H.G. (1995). Natural product flavor compounds as potential antimicrobials, insecticides, and medicinals. Agro-FoodIndustry Hi-Tech. 6: 19-23.

Domans, H.J.D. and Deans, S.G. (2000). Antimicrobial agents from plants: antibacterial activity of plant volatiles oils. Journal of Applied Microbiology. 88: 308-316. 
Dubey, S., Upadhyay, P.D. and Tripathi, S.C. (1991). Fungitoxic and insect repellant efficacy of some spices, Indian Phytopathol. 44: 101-105.

El-Kady, I.A. and Moubasher, M.H. (1982). Toxigenicity and toxin of Stachybotrys chartarum isolates from wheat straw samples in Egypt. Experimental Mycology 6: $25-31$.

El-Shami, M.A., Fadl, F.A., Sirry, A.R. and El-Zayat, M. M. (1985). Antifungal property of garlic, clove juice compared with fungicidal treatment against Fusarium with watermelon. Egyptian Journal of Phytopathology.17: 55-62.

Farag, R.S., Daw, Z.Y. and Abo-Raya, S.H. (1989). Influence of some spice essential oils on Aspergillus parasiticus growth and production of aflatoxins in a synthetic medium, J Food Sci. 54: 7476.

Gimeno, A. (1979). Thin-layer chromatographic determination of aflatoxins, ochratoxins,

sterigmatocystin, zearalenone, citrinin, $\mathrm{T}-2$ toxin, diacetoxyscirpenol, penicillic acid and penitrem "A". J. Assoc. Off. Anal. Chem. 62: (579- 585).

Hitokoto, H., Morozumi, S., Wauke, T., Sakai, S. and Kurata, H. (1980): Inhibitory effects of spices on growth and toxin production of toxigenic fungi. Appl. Environ. Microbiol.39: 818-822.

Juglal, S., Govinden, R. and Odhav, B. (2002). Spices oils for the control of co-occuring mycotoxinproducing fungi. Journal of Food Protection. 65:638-687.

Köhl, J., Postma, J., Nicot, P., Ruocco, M. and Blum, B. (2011).
Stepwise screening of microorganisms for commercial usein biological control of plant-pathogenic fungi and bacteria. Biological Control, 57: $1-12$.

Leontopoulos, D., Siafaka, A. and Markaki, P. (2003). Black olives as substrate for Aspergillus parasiticus growth and aflatoxin B1 production. Food Microbiology. 20(1): 119-126.

Mabrouk, S.S. and EL-Shayeb, M.M.A. (1981). Inhibition of aflatoxin formation by some spices. Z. Lebesm-Unters. Forsch. 71: 344-347. Cited in: Chem. Abst. 94, 14091h (1981).

Mari, M., Neri, F. and Bertolini, P. (2007). Novel approaches to prevent and control postharvest diseases of fruit. Stewart Postharvest Review, Redhill.3:1-7.

Mostafa, E.M. (1990). Mycoflora and mycotoxins of some spices. M. Sc. Thesis, Botany Dept., Faculty of Science, Assiut University, Egypt.

Radhakrishanan-Sridhar, S. and VelusamyRajaopal, R. (2003). Antifungal activity of some essential oils. Journal of Agriculture and Food Chemistry. 51:7596-7599.

Rasooli, I., Rezaei, M.B. and Allameh, A. (2006). Growth inhibition and morphological alterations of Aspergillus niger by essential oils from Thymus eriocalyx and Thymus x-porlock. Food Control. 17: 359-364.

Ristori, C.A., Pereira, M.S. and Gelli, D.S. (2002). O efeito da pimenta do reino moída frente a contaminação in vitro com Salmonella rubislaw. Revista do Instituto Adolfo Lutz. 61: 131-133. 
Schmitz, S., Weidenbgner, M. and Kunaz, B. (1993). Herbs and spices as selective inhibitors of mould growth, Chemie, Mikrobiologie Technologie der Lebensmitte.15: 175 177.

Scott, P.M., Lawrence, J.W. and Van Walbeak, W. (1970). Detection of mycotoxins by thin-layer chromatography: Application to screening of fungal extracts. Appl. Microbiol 20: 839 - 842.

Sharma, R.R., Singh, D. and Singh, R. (2009). Biological control of postharvest diseases of fruits and vegetables by microbial antagonists: A review. Biological Control.50(3): 205-221.

Smid, E.J. and Gorris, L.G.M. (1999). Natural antimicrobials for food preservation. In: Rahman, M. S. (Ed.), Handbook of Food Preservation. Marcel Dekker, New York, pp. 285308.

Souza, E.L., Stamford, T.M.S., Lima, E.O. Trajano, V.N. and José Maria Barbosa Filho, J.M.B. (2005). Antimicrobial effectiveness of spices: an approach for use in food conservation systems. Brazilian archives of Biology and Technology. 48: 549-558.

Thyagaraja, N. and Hosono, A. (1996). Effect of spice extract on fungal inhibition. Lebensmittel- Wissenchaft und-Technologie. 29: 286288.

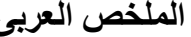

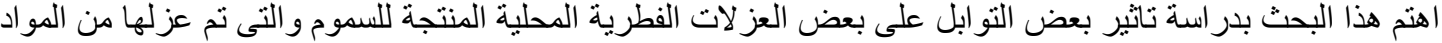

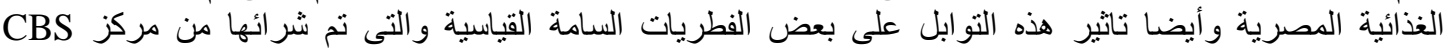

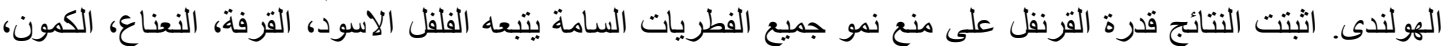

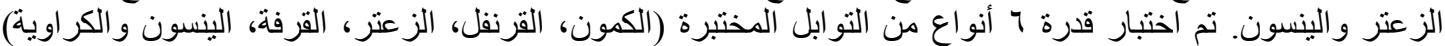

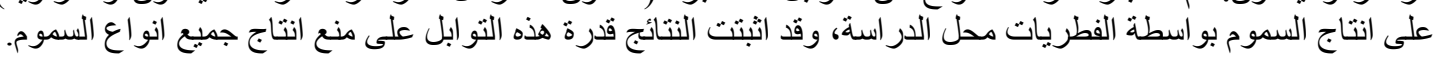

